\title{
Permeability of Broken-Cell Foamed Materials
}

\author{
J. P. Bosscher \\ Calvin College, Grand Rapids, Mich. \\ F. E. Fisher \\ Dept. of Mechanical Enginecring \\ University of Michigan \\ Ann Arbor, Mich.
}

(Received April 10, 1970)

\section{Introduction}

he flow of fluids through open-cell foamed materals has been investigated previously by Gent and Rusch (1) and useful relationships, relating foam physical parameters and flow characteristics determined. Fluid flow through broken-cell foams, created by crushing foams possessing discrete cells so as to rupture inter-cell walls, has not been previously treated in detail. Such a study is of interest inasmuch as many foams cannot be produced in open-cell form and are, therefore, fabricated with broken cell-walls which act to influence flow between pores. The additional energy dissipation available when fluid flows through such media make it of interest in a variety of shock and vibration absorption applications. A study of the permeability of such broken-cell foamed materials has, therefore, been made and is herein described.

\section{Analytical Considerations}

Since crushed-cell foams are conceived as opencell foams with inter-cell valves between cells, an idealized in-channel, rectangular flapper valve (see Figure 1) is proposed. Assumptions made to idealize the situation are:

1. Rectangular flapper valve and passage

2. Flapper valve angle of deflection small-change in pressure on valve uniform across valve surface

3. Fluid experiences only small density changes across valve

4. Flowing gas can be considered an ideal gas with constant specific heat.

Analysis leads to the following expressions for the pressure differential across the flapper valve.

$$
\Delta \mathrm{P}=\left[\frac{\mathrm{k}-1}{\mathrm{k}}\right]^{1 / 5}\left[\frac{\mathrm{Et}^{3} \rho^{1 / 4}}{\mathrm{l}_{\mathrm{f}}^{7 / 2} \mathrm{~b}_{\mathrm{f}}^{1 / 2}}\right]^{2 / 5} \mathrm{~A}_{\mathrm{c}}^{2 / 5} \mathrm{~V}_{\mathrm{c}}^{2 / 5}
$$

where $k$ is the ratio of constant pressure specific heat to the constant volume specific heat for the flowing gas, $\mathrm{E}$ is the elastic modulus of the valve material, $\rho$ is the density of the flowing fluid, $A_{c}$ is
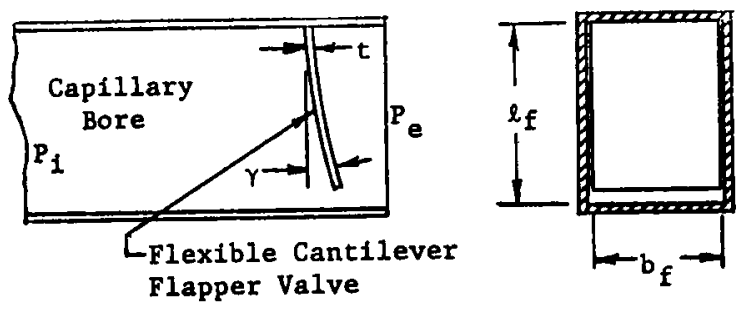

Figure 1. Flapper valve geometry.

the capillary bore area, and $V_{c}$ is the velocity of the fluid in the capillary. If it is further assumed that the average cell is square of height and of height and width $l_{r}$ and

width $l_{t}$ and the factor $\left[\frac{k-1}{k}\right]^{t / 5}$ is approxi-

mately unity, the relation becomes,

$$
\Delta \mathrm{P}=\left[\frac{\mathrm{Et}^{3} \rho^{1 / 4}}{\mathrm{l}_{\mathrm{f}}^{4}}\right]^{4 / 5} \mathrm{l}_{\mathrm{s}}^{4 / 6} \mathrm{~V}_{\mathrm{c}}^{* / / \pi}
$$

Further manipulation gives,

$$
\Delta \mathbf{P}=\left[\frac{\mathrm{Et}^{3} \rho^{1 / 1}}{\mathrm{l}_{\mathrm{s}}^{3}}\right]^{1 / 4} \mathrm{~V}_{\mathrm{c}}=
$$

If the length over which the pressure drop occurs is taken as one cell length, the pressure gradient becomes,

$$
\frac{\Delta \mathrm{P}}{\Delta \mathrm{X}}=\left[\frac{E^{4 / 5} \mathrm{t}^{12 / 5} \rho^{1 / 5}}{\mathrm{I}_{\mathrm{f}}^{7 / 5}}\right] \mathrm{V}_{\mathrm{c}}^{2 / 5}
$$

Further simplification leads to the following approximation for the pressure gradient due to inter-cell valves.

$$
\frac{\Delta \mathrm{P}}{\Delta \mathrm{X}}=\frac{E \mathrm{t}^{2.6} \rho^{1 / 4}}{l_{\mathrm{r}}} \mathrm{V}_{\mathrm{c}}^{1 / 2}
$$

Gent and Rusch (1) indicate that the pressure gradient required to maintain fluid flow at a velocity $\mathrm{V}$ through a porous medium may be given as follows: 


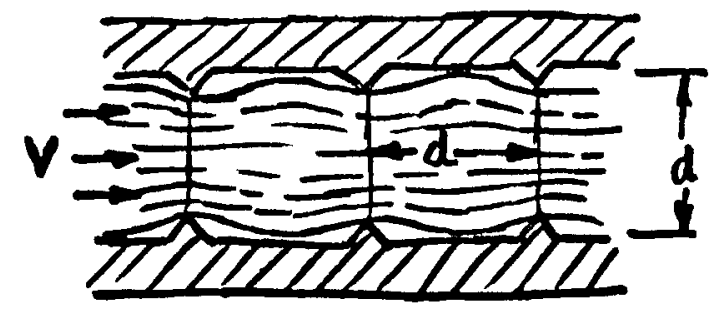

Figure 2. Modified straight-tube model.

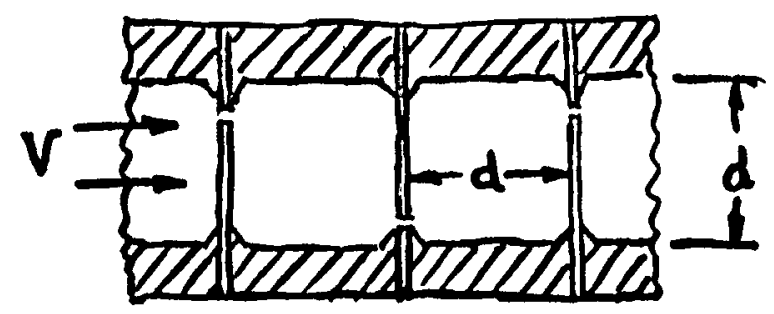

Figure 3. Modified straight-tube model with valves.

$$
\frac{\partial \mathrm{P}}{\partial \mathrm{X}}=\frac{\eta}{\mathrm{K}} \mathrm{V}+\frac{\rho}{\mathrm{B}} \mathrm{V}^{2}
$$

where $\eta$ is the viscosity and $\rho$ the density of the fluid, and $K$ and $B$ are coefficients characteristic of the medium. The coefficient $K$ is known as the permeability of the medium and has dimensions of area. For a simple model, consisting of an array of straight tubes of circular cross-section and diameter $\mathrm{d}$ it can be shown that,

$$
\mathrm{K}=\frac{\mathrm{d}^{2}}{32}
$$

The $\mathrm{V}^{2}$ term in Equation (5) reflects the inertial forces developed in turbulent flow caused by expansion-contraction losses in irregular flow channels. Considering a modified straight-tube model (see Figure 2) containing a series of orifices, it can be shown (1) that B is a coefficient characteristic of orifice shape, nature of the flow, and the ratio $D^{\prime}$ of the diameter of the tube to that of the orifices. For well-rounded orifices, with one orifice for each length along the tube equal to $d$, the tube diameter and for $D^{\prime}$ not much greater than unity, B is closely approximated by

$$
\frac{1}{\mathrm{~B}}=\frac{1}{2 \mathrm{~d}}\left[\left(\mathrm{D}^{\prime}\right)^{2}-1\right]^{2}
$$

This treatment of the $\mathrm{V}^{2}$ term of the pressure gradient relation does not provide for velocity distribution across the tube cross-section or the effect of one constriction upon the pressure drop developed at the next. Experimental results (1) for a series of foams over a large range of flow rates show values of B to be "accurately constant".

Assuming results of flapper valve flow relations and experiments to be valid, the introduction of such a valve (see Figure 3 ) at each orifice of the

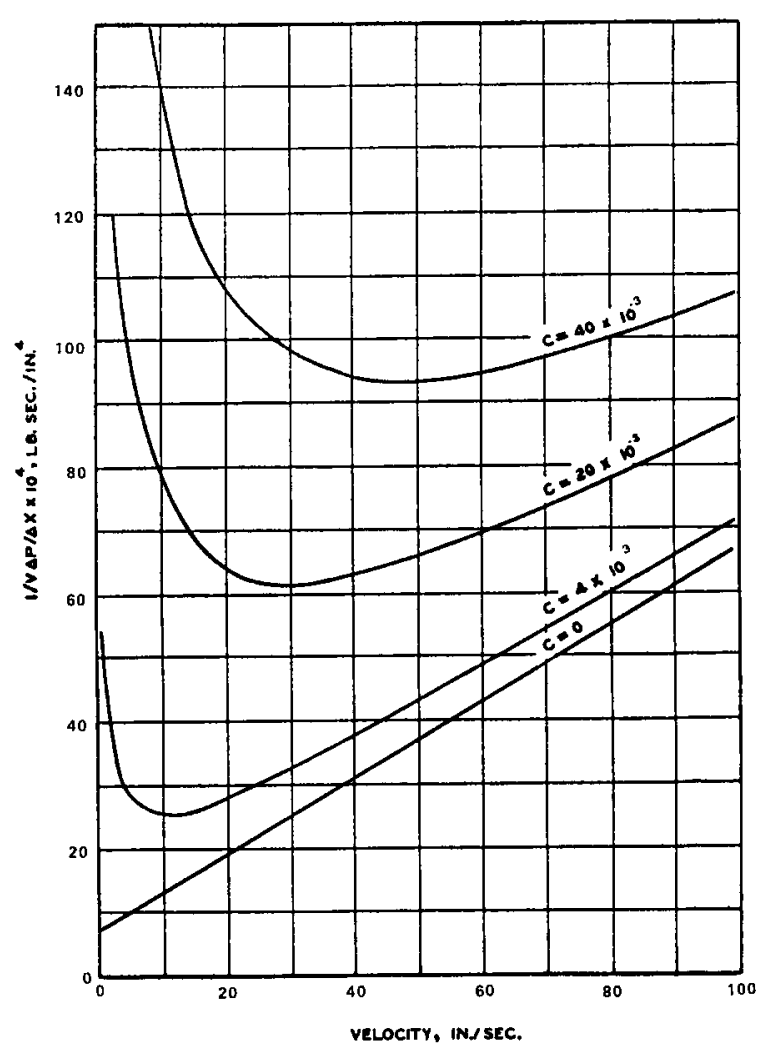

Figure 4. Pressure gradient versus flow velocity utilizing assumed coefficient values in the suggested equation for crushed-cell foam.

$$
\left(\partial \mathrm{P} / \partial \mathrm{X}:=7.5 \times 10^{-4} \mathrm{~V}+6.0 \times 10^{-5} \mathrm{~V}^{2}+\mathrm{CV}^{1 / 2}\right)
$$

modified straight-tube model should result in an additional term in Equation (5). This term would properly consist of relation similar to the right side of Equation (4). Inserting this term in Equation (5) gives,

$$
\frac{\partial \mathrm{P}}{\partial \mathrm{X}}=\frac{\eta}{\mathrm{K}} \mathrm{V}+\frac{\rho}{\mathrm{B}} \mathrm{V}^{2}+\mathrm{CV}^{1 / 2}
$$

where $\mathrm{C}$ includes the parameters in Equation (5) other than $V_{c}^{1 / 2}$. If Equation (8) is divided throughout by $\mathrm{V}$ we have,

$$
\frac{1}{\mathrm{~V}} \frac{\partial \mathrm{P}}{\partial \mathrm{X}}=\frac{\eta}{\mathrm{K}}+\frac{\rho}{\mathrm{B}} \mathrm{V}+\mathrm{CV}^{-1 / 2}
$$

A plot of Equation (9) with $C$ set equal to zero results in a linear graph of slope $\rho / B$ and intercept $\eta / \mathrm{K}$. Experimental results (1) are in good agreement for open-cell foams.

Assuming typical values of $\rho / \mathrm{B}$ and $\eta / \mathrm{K}$ for the flow of air through open-cell foams $(\mathrm{C}=0)$ results in the straight line plotted in Figure 4. Employing a series of values for the coefficient $\mathrm{C}$ of the halfpower term in Equation (9) results in the addition of a fillet-type curve to the straight-line plot. This so-called "fillet-type" curve is seen to occur between the zero velocity vertical line and the sloping line for $\mathrm{C}$ equal to zero-the value of the radius of the 


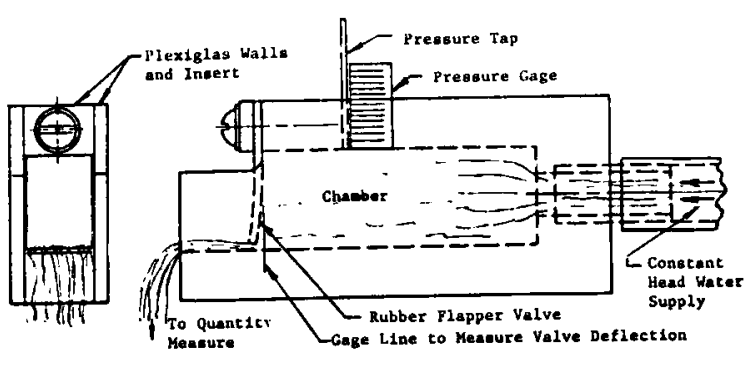

Figure 5. Flapper valve flow mixture.

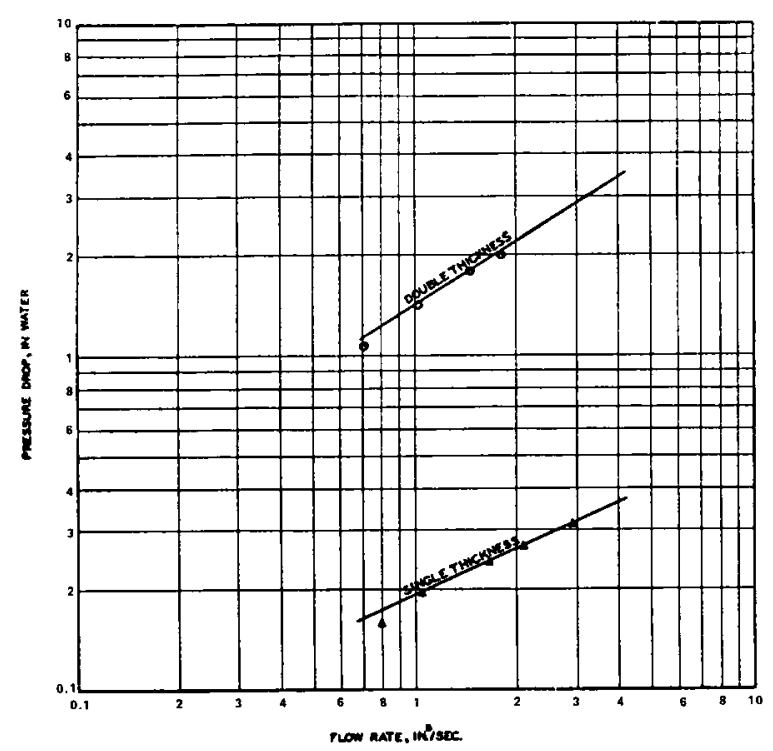

Figure 6. Pressure drop versus flow rate in flapper valve flow testing.

"fillet" growing with an increase in the coefficient C.

\section{Flapper Valve Flow Testing}

To check the relations derived for flow through a flapper-type valve a flow fixture (see Figure 5) was produced and tests conducted. As can be seen by reference to Figure 6, the slope of the flow rate versus pressure drop log-log plot possesses a value of about 0.47 as opposed to the derived value of 0.40 as given in Equation (1) and (2).

Two values of valve thickness were tested, as shown in Figure 6. Isolating the effect of thickness indicated that the derived relation checked reasonably, but not exactly-5.4 was predicted and 6.7 was the actual number. Possible reason for the disparity may relate to the fact that the double thickness valve was constructed by cementing two single thicknesses together creating a member somewhat stiffer in the center due to the cemented construction.

The value of the intercept in Figure 6, upon correction from inches of water to pounds per square inch, was found equal to .008 . This compares favorably with the value of .010 . found by

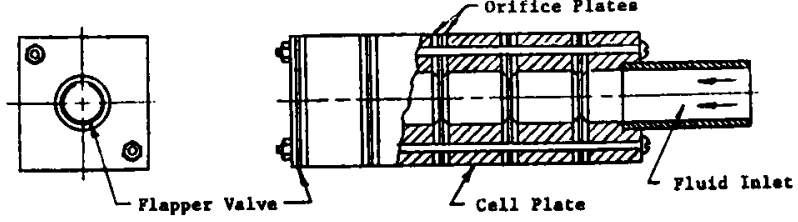

Figure 7. Foam simulator.

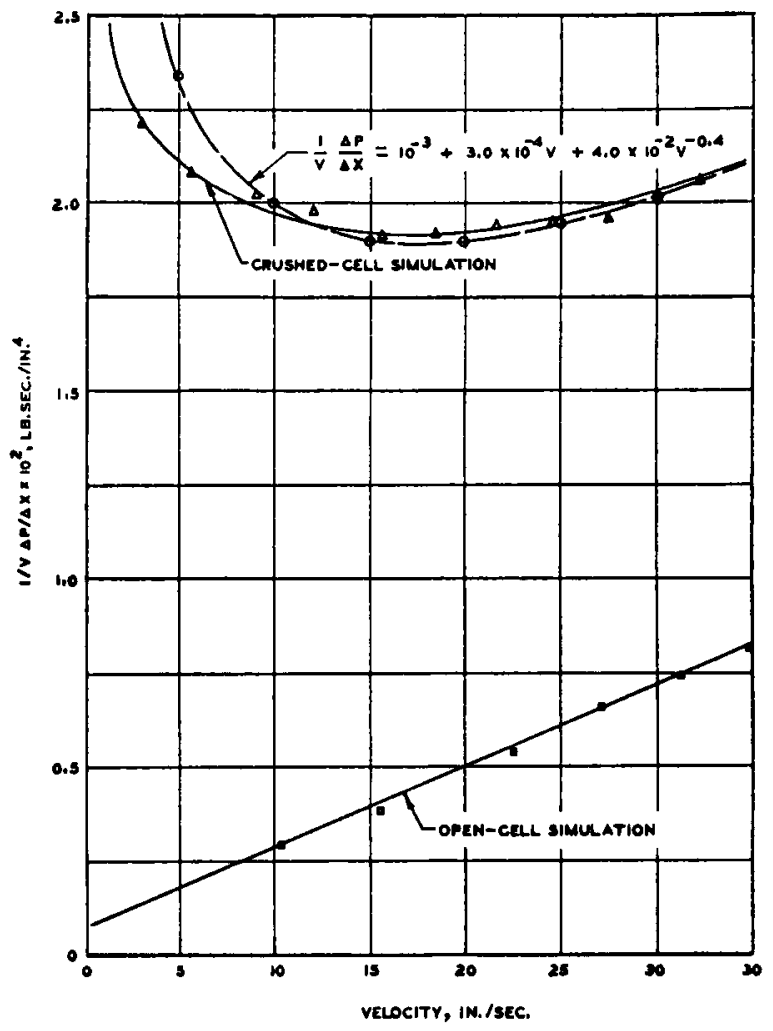

Figure 8. Pressure gradient versus flow velocity in simulated foam testing utilizing water as fluid.

substituting actual physical values in the derived relations.

\section{Fluid Flow Through Real and Simulated Foams}

To compare pressure gradient relations suggested for crushed-cell materials, experiments were conducted employing fluid flow through real and simulated foams.

The simulated foam utilized consisted of a tube with orifices spaced at regular intervals equal to the internal diameter of the tube similar to the model previously suggested. As illustrated in Figure 7, flapper valves can be inserted between orifice plates, thus allowing a conversion from opencell simulation-a shift which is impossible to effect in actual foams. The simulator was tested in three forms:

1. without valves using water as the flowing fluid 


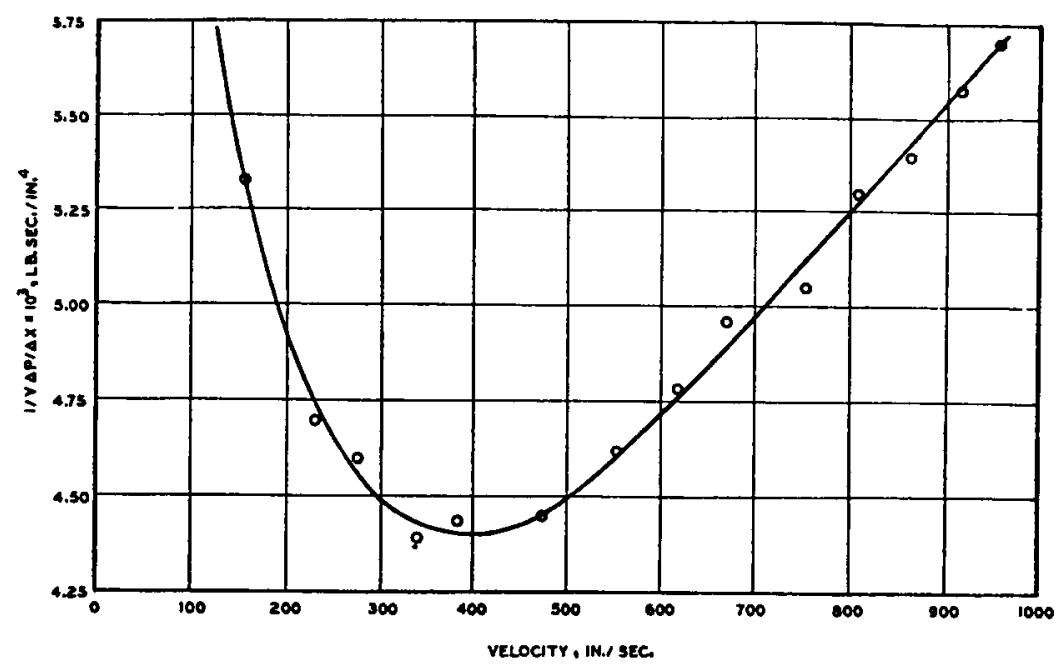

Figure 9. Pressure gradient versus flow velocity in simulated foam testing utilizing air as fluid.
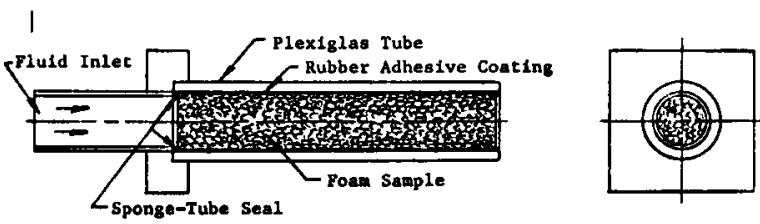

Figure 10. Foam sample mounted for flow testing.

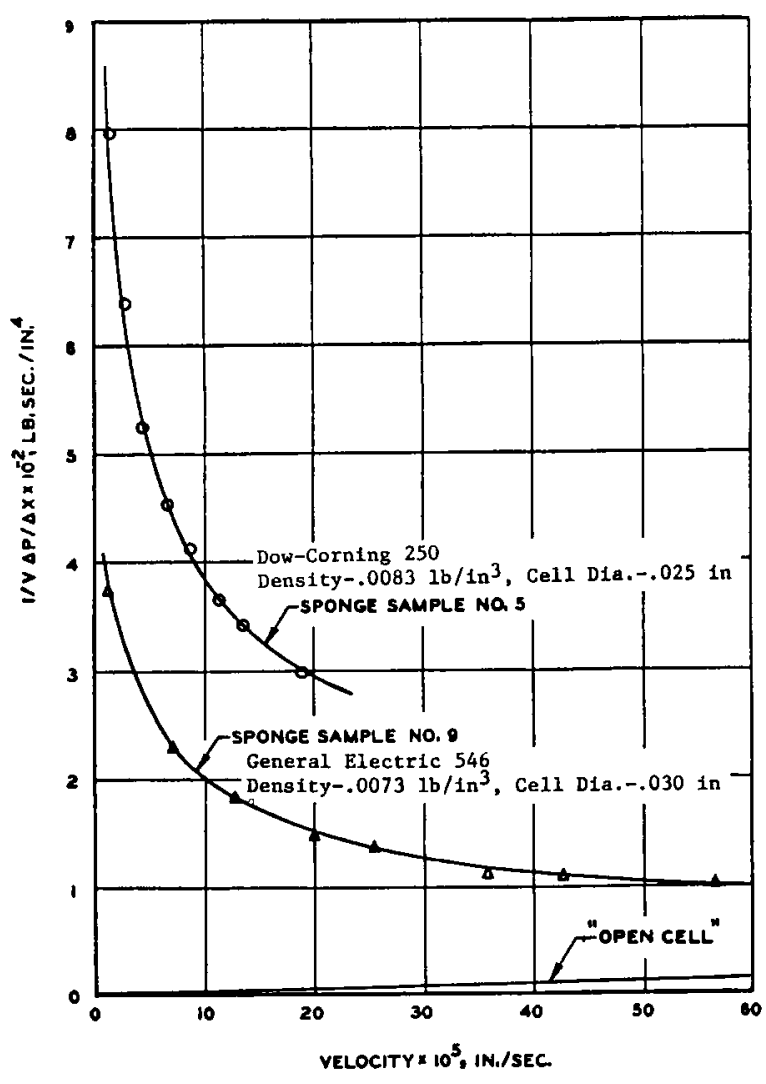

Figure 11. Pressure gradient versus flow velocity in actual foam testing utilizing water as fluid.
2. with intercell valves using water as the flowing fluid

3. with intercell valves using air as the flowing fluid

Results of such tests are illustrated in Figures 8 and 9. Reference to the "crushed-cell" simulation (with flapper valves) results (reported in Figures 8 and 9) reveals the fillet-curve effect related previously (see Figure 4) to the half-power term. In addition, the open-cell simulation shows the expected straight line $(\mathrm{C}=0)$ behavior and the appropriate relation (eventually assymptotic) to the crushed-cell simulation curve.

Flow through actual crushed-cell foam was included in the experimental program. As illustrated in Figure 10, cylindrical samples of foams were inserted in tubes and water was caused to flow down the axis of the samples. Results of such tests are illustrated in Figure 11. Once again the fillet-curve result predicted by the half-power velocity effect is evidenced in the graphical results. Straight line open-cell results based on estimated values are plotted for contrast.

\section{General Conclusions}

Results in all flow experiments for actual and simulated foam seem to include the partial power velocity effect for crushed-cell situations. Most vital proof is indicated by the results reported in Figure 11 where the flow of water through two actual foams is reported graphically. Both foams show sharp increases in the $\frac{1}{V} \frac{\Delta P}{\Delta \mathrm{X}}$ parameter as the velocity approaches zero, as predicted by Equation (9). In addition, the curve for the G.E. 546 sample shows the higher velocity behavior predicted by theory; i.e., it is moving toward becoming assymptotic to the presumed "open-cell" line as velocities increase. The higher pressures began to distort the 
Dow-Corning 250 sample in the tube, and therefore, higher velocities could not be investigated. Expanded investigations to firmly establish the proper constituents of the half-power term coefficient (guided by the theoretical treatment) and, in addition, to carefully evaluate the exponent of this term, should be most instructive.

\section{Literature Reference}

1. A.N. Gent and K.C. Rusch, J. Cellular Plastics, 2,46 (1966).

\section{Acknowledgments}

The author acknowledges with thanks the foam samples prepared for this investigation by the Detroit Silicone Rubber Company, the Dow-Corning Corporation, and the DuPont Company. He also wishes to thank the National Science Foundation and the Michigan Institute of Science and Technology for the provision of fellowships, during the tenure of which this work was performed.

\section{List of Symbols}

$\Delta \mathrm{P}=$ pressure differential

$\mathrm{k}=$ ideal gas specific heat ratio

$\mathrm{E}=$ elastic modulus of valve material

$\mathrm{t}=$ valve thickness

$\rho=$ fluid density

$A_{c}=$ capillary bore area

$\mathrm{V}_{\mathrm{c}}=$ fluid velocity in capillary

$l_{\mathrm{f}}=$ flapper valve length

$\mathrm{b}_{\mathrm{t}}=$ Happer valve width

$\partial \mathrm{P} / \partial \mathrm{X}=$ pressure gradient causing flow

$\mathrm{V}=$ flow velocity

$\eta=$ viscosity of flowing fluid

$\mathrm{K}=$ permeability constant

$\mathrm{B}=$ flow resistance constant

$\mathrm{A}=$ cross-sectional area normal to flow direction

$\mathrm{d}=$ average diameter tubes or cells

$\mathrm{D}^{\prime}=$ ratio of diameter of the flow channel to that of the orifices in the model

$\mathrm{C}$ = arbitrary coefficient for half-power velocity term
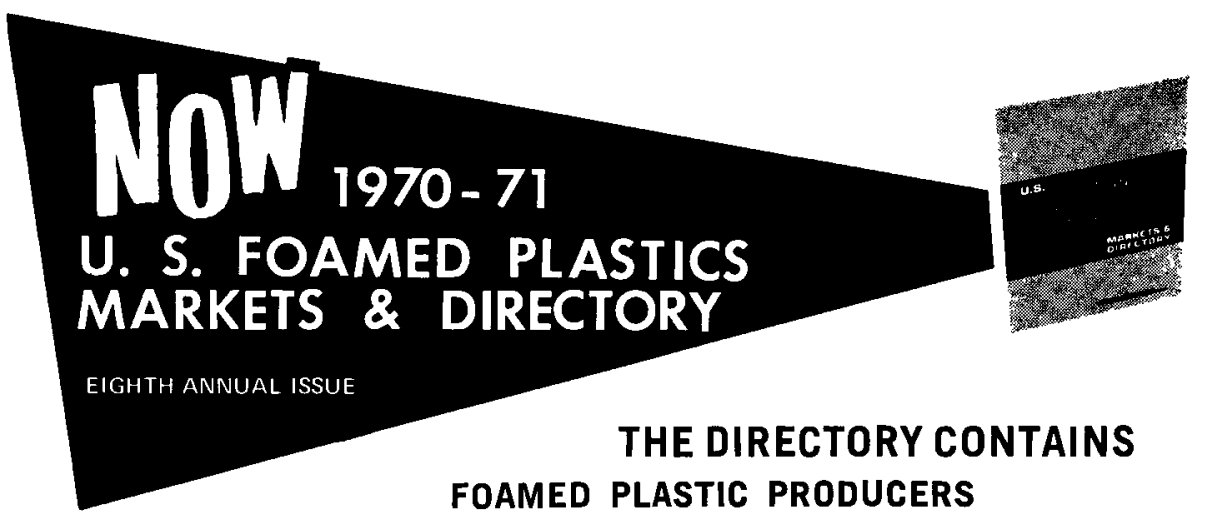

$\$ 15$

Raw material suppliers

Foam producers

Processors and fabricators

Laminators (garment)

Machinery manufacturers

Distributors

\section{... Hundreds of producers and users of foamed plastics \\ ... A guide to markets in this fast-growing field}

Complete Information

The Directory covers all foamed plastics and contains the most complete listing of foamed plastic companies ever assembled, including addresses, phone numbers, key personnel, kinds of foam handled, and description of manufacturing activity.

Marketing Guide and Statistical Section

The 1970-71 edition contains a complete marketing and statistical section covering all commercial foam, including PVC foam. It has many tables which give formulations, starting with raw materials, and developing the manufacturing economics to show what profit-ability is for various vol. ume manufacturing levels. This marketing section alone is worth the price of having this publication in your personal library. Add to this, for the first time a classification of foam companies by the end-use product they make, and you can see why this publication is the most complete manufacturing and selling guide to the foam industry, worldwide.

Future Foamed Plastic Growth Patterns

This year's directory \& marketing guide will give you the guidelines you need for planning your future moves in the foamed plastics industry. For each of the major commercial foamed plastics, current markets, future market potential, and end use patterns and growth rates are given. Projected economic analyses of each foam marketing area will enable you to predict profitability and market penetration, and permit comparison of commercial products competitive with foamed plastics. 